\title{
Homeopathy and the science of high dilutions: when to believe the unbelievable
}

\author{
Paolo Bellavite ${ }^{1}$ and Lucietta Betti ${ }^{2}$ \\ ${ }^{1}$ Department of Pathology and Diagnostics, University of Verona, Italy \\ ${ }^{2}$ Department of Agroenvironmental Sciences and Technologies, University of Bologna, Italy
}

\begin{abstract}
"How small must be the dose of each individual medicine, homeopathically selected for a case of disease, to effect the best cure? [...] not the work of theoretical speculation... Pure experiment, careful observation of the sensitiveness of each patient, and accurate experience can alone determine this in each individual case."

C.F.S. Hahnemann, Organon, par. 278
\end{abstract}

In 1988, an editorial in journal Nature [1] titled "When to believe the unbelievable" observed that the famous high-dilution experiments by Benveniste's group [2] had no physical basis and suggested that "prudent people should, for the time being, suspend judgment." However, judgment was not suspended at all, but in the following weeks the authors were condemned and ridiculed for the alleged lack of replication of their findings. Twenty-four years later, although the idea that solutions beyond Avogadro's constant may exhibit biological and pharmacological activity remains "unbelievable" to the common sense, things are changing dramatically. Several groups of researchers from countries all over the world are devoted to this puzzling subject, which has weighty implications not only for pharmacology (homeopathy) but also for biology, physics and the environmental sciences. The XXVI meeting of GIRI (International Research Group on Very-low dose and High-Dilution Effects), scheduled to take place in Florence on September 20-22, 2012, will present a growing body of evidence attesting to the effects of high dilutions (HD). The program is organized around four main axes: physicochemical features of HD, studies in plants and the field, clinical and veterinary evidence, and laboratory models.

The study of HD is a new interdisciplinary field almost inextricably linked to the two-century old problem on the nature of homeopathic medicines. Notwithstanding its widespread practice and the high levels of patient satisfaction, homeopathy gives rise to intense skepticism, because its medicines are subjected to a process of serial dilution that results in extremely low (often non-measurable) levels of active principles. Skepticism is only one short step away from ridicule, which would be justified were homeopathic medicines be nothing besides high dilutions. However, the preparation of HD also involves agitation, which introduces mechanical energy and strong turbulence into the system. This step might impart a nano-heterogenous structure to water by means of phenomena such as coherence, epitaxy, temperature-pressure alterations, and formation of nanobubbles containing gaseous inclusions of oxygen, nitrogen, carbon dioxide, silica, and possibly also the source materials of medicines. The existence and nature of clathrate-like hydrate nanostructures formed during the dilution/agitation process might be explained by cluster science, according to which the chemical reactivity of different geometrical structures of clusters of a same chemical species may differ [3]. HD are not like ordinary bulk drugs, and recent evidence suggests they should be considered as colloidal solutions of nanoparticles of the source material, the solvent, and possibly also the container's (e.g. silica) $[4,5]$. This view agrees with observations performed with low-temperature thermoluminescence spectroscopy by Rey [6], and results obtained with UV spectroscopy, biocrystallization and the droplet evaporation method will be presented at the XXVI GIRI meeting. These methods are considered to be promising tools for the investigation of subtle changes in the structure of water and the effects of homeopathic preparations, particularly in plants 
and biological fluids. Also new applications of DNA microarray technology to the investigation of homeopathic drugs will be discussed.

Elia's group in Naples gathered an impressive amount of experimental evidence on "anomalous states of water". These authors studied the electrical conductivity, heat of mixing with acid or basic solutions, and $\mathrm{pH}$ of water, and found that all three were able to detect changes in the structure of water in space-time. This phenomenon might be accounted for by a common working hypothesis, i.e., formation of dissipative structures. Water is a complex liquid capable of self-organization induced by mechanical and/or electromagnetic perturbations even when small. Increase of environmental entropy due to dissipation, and decrease due to formation of local orders still amount to overall increase in entropy, whereby formation of local structures in water occurs spontaneously. In the liquid phase, such structures are able to remain in a far-from-equilibrium state through dissipation of radiant energy drawn from the environment, whereas in the solid phase they are able to retain their properties indefinitely without dissipation. When sufficient water becomes available, these nanostructures are able to exploit radiating energy from the environment and thus revert to the far-from-equilibrium state. The existence of these aqueous nanostructures in solid phase represents a novel and totally unexpected phenomenon that is met in clinical practice whenever homeopathic globules are dissolved in the patient's mouth: the aggregates are restored to the liquid phase and thus recover their ability to dissipate environmental energy to maintain their far-from-equilibrium state. Under such conditions, they might exert their therapeutic action as dissipative structures.

The biological mechanism(s) underlying the regulatory processes affected by HD must yet be elucidated. Various lines of investigation suggest that HD might affect some subtle and early levels of signal transduction and/or genetic expression. Changes in the structure of water induced by HD might influence a crucial layer of water surrounding the cells, and thus also signal detection and transduction. Further studies are needed to confirm whether this hypothesis applies to the effects of HD observed in humans and plants.

One thing is certain: HD pharmacology challenges the dose-response dogma. Rather than an exception, nonlinearity between dose and response is the rule in biological systems. The occurrence of dual effects (both stimulatory and inhibitory) caused by a same agent in different doses or at different times was described in various experimental systems, and is often referred to as "hormoligosis", or "hormesis". So-called "paradoxical pharmacology" is a related phenomenon belonging with the in vivo effects of drugs, whereby the acute and chronic actions of drugs often exhibit opposite effects. This is particularly true for receptor-mediated events. The susceptibility of a complex system is greatly enhanced close to a phase transition or critical point, and under such conditions, when random energy ("noise") is added, even minute perturbations push the system over the energy barrier. This phenomenon, called stochastic resonance, may represent the physical explanation of the effect of agitation in the preparation of HD. This same mechanism may also operate in vivo, where the stochastic resonance provided by noise from biochemical reactions may amplify the effect of the small but highly specific information supplied by the drug inside the living organism. Interestingly, the results obtained by Jäger's group comparing the response to homeopathic preparations of duckweed and yeast agree with the hypothesis that more complex organisms show stronger reactions to homeopathic medicines compared to less complex organisms. Others and we intuitively predicted that the "potency" increase induced by serial dilution might have a physical basis grounded on the theory of self-similarity and fractal structures formation in serial dilutions (seen as mathematical iteration processes).

Further development of basic research is highly desirable, and several reports to be presented at the GIRI meeting suggest that cell- and plant-based bioassays may be suitable tools for this purpose. Botanical bioassays seem particularly suitable in this regard, because they allow for large numbers of experimental replications. One major challenge basic research must meet is the development of test systems able to yield consistent results. The hindrance represented by subjective evaluation is avoided by means of the systematic use of: a) spectroscopic quantitative methods; b) multiple intra-series and inter-series replications; c) coded 
test samples; d) highly pure compounds and parallel comparisons with control pure-water dilutions; e) mechanical shaking methods with standardized duration and frequency. A variety of protocols and different experimental conditions in terms of types of dilution/shaking procedures and solvent used should be explored. Research on extremely sensitive systems and very high dilutions of substances suggests that trace elements, container materials, storage duration and shaking methods may influence the results. Therefore, suitable water controls prepared in an identical manner and subjected to a same storage time must be used. As a function of these considerations and the controversial nature of the investigated subject, independent replications are crucial to establish stable models that might be used by different investigators worldwide.

For two centuries, homeopathy and science were considered to be two opposing and conflicting fields. Now things are changing, as scientific evidence begins to support many homeopathic tenets, and the homeopathic world increasingly stimulates science to investigate previously under-evaluated and little understood subtle phenomena. For 26 years, GIRI has been promoting a fruitful dialog for the sake of furthering true science, i.e., science free from ideological barriers and preconceptions. Novel evidences have quite often disrupted scientific "dogmas", or previously dismissed phenomena grew in importance within new conceptual frameworks. The unusual properties of HD, which deserve further investigation, are potentially relevant not only to homeopathic pharmaceutical science, but also to agriculture ("agrohomeopathy"), the environmental sciences and the future of humankind as a whole.

\section{References}

[1] Opinion. When to believe the unbelievable. Nature. 1988; 333(787): 6176.

[2] Davenas E, Beauvais F, Amara J, Robinson M, Miadonna A, Tedeschi A, Pomeranz B, Fortner P, Belon P, Sainte-Laudy J, Poitevin B, and Benveniste J, Human basophil degranulation triggered by very dilute antiserum against IgE. Nature. 1988; 333: 816-818.

[3] Roy R, Tiller W, Bell IR, and Hoover MR, The structure of liquid water. Novel insights from materials research; potential relevance to homeopathy. Mat.Res.Innovat. 2005; 9: 98-103.

[4] Chikramane PS, Suresh AK, Bellare JR, and Kane SG, Extreme homeopathic dilutions retain starting materials: A nanoparticulate perspective. Homeopathy. 2010; 99: 231-242.

[5] Upadhyay RP, Nayak C. Homeopathy emerging as nanomedicine. Int J High Dilution Res. 2011 [cited 2012 Aug 5]; 10(37): 299-310. Available from:http://www.feg.unesp.br/ ojs/index.php/ijhdr/article/view/525/551

[6] Rey L, Can low-temperature thermoluminescence cast light on the nature of ultra-high dilutions? Homeopathy. 2007; 96: 170-174.

\section{(c)) BY-NC-ND Licensed to GIRI}

How to cite this article: Bellavite P, Betti L. Homeopathy and the science of high dilutions: when to believe the unbelievable [editorial]. Int J High Dilution Res [online]. 2012 [cited YYYY Month dd]; 11(40): 107-109. Proceedings of the XXVI GIRI Symposium; 2012 Sep 20-22; Florence (Italy). GIRI; 2012; Available from: http://www.feg.unesp.br/ ojs/index.php/ijhdr/article/view/614/622 